affluence but an unnecessary burden on the disadvantaged'. ${ }^{5}$

Of growing interest is the evidence suggesting a direct relationship between oral health and diseases such as cardiovascular disease and diabetes. This is potentially of great public health significance, as oro-dental disease is largely preventable, and in many instances readily treatable. If oral health is to be recognised within the broader health system as having an important part to play in improving the health of our community, it is imperative that this relationship between oral health and other diseases be examined more closely.

To this end, and beginning with this edition of the Public Health Bulletin, a series of articles will examine current and emerging issues in oral health. These issues are both exciting and challenging, not only for the oral health profession, but also for medical and public health professionals. Over four issues, the Bulletin will examine topics such as the oral manifestations of child neglect; the influence of oral health on the general health of the aged and those with special needs; oral carcinoma; and the impact of HIV-AIDS on oral health. The future workforce required to meet the changing patterns of disease resulting from our aging population; the economic impact of oral disease; and research trends will also be explored.

This edition highlights two important areas. The first article examines the relationship between periodontal disease and systemic health, with particular reference to pregnancy, diabetes and cardiovascular disease. The second explores the impact on oral health of xerostomia (dry mouth), the most common cause of which is medication.

\section{REFERENCES}

1. Improving Dental Health in Australia. National Health Strategy Background Paper No. 9. Canberra: AGPS, 1992. ISBN 0642174687.

2. Australia, Parliament 1998, Inquiry into public dental services, Senate Community Affairs References Committee Report, Parliamentary Paper. Canberra: Commonwealth of Australia, 1998.

3. Australian Bureau of Statistics 1995, National Health Survey, Cat. no. 4364.0, ABS, Canberra.

4. Mathers C, Pen MR, Carter C, Stevenson C. Health system costs of diseases and injury in Australia 1993-94, Health and Welfare Expenditure Series No. 2, Canberra: Australian Institute of Health and Welfare, 1998.

5. Kickbusch I. Self-care in health promotion. Soc Sci Med 1989; 29(2): 125-130. F

\title{
PERIODONTAL DISEASES AND SYSTEMIC HEALTH: ASSOCIATIONS, DIRECTIONS, IMPLICATIONS
}

\section{Barbara Anne Taylor}

Head, Department of Periodontics

United Dental Hospital of Sydney, Surry Hills

Recent findings that suggest that periodontal disease may be an independent risk factor for a number of significant systemic diseases, including cardiovascular diseases, are so important that they have been referred to as 'the inversion of a paradigm'. ${ }^{1}$ While it is well known that a number of diseases and normal physiological states are associated with an increase in the risk of periodontal disease, until now we have not recognised that the chronic infection and ulceration in periodontal disease could affect systemic health beyond certain limited and welldefined instances, such as bacterial endocarditis. This article describes the association between periodontal disease and systemic health, in particular the evidence for an effect in pregnancy, diabetes and cardiovascular disease; and the implications of this for public health.

\section{THE PERIODONTAL DISEASES}

The periodontal diseases are a family of chronic inflammatory diseases, including gingivitis and periodontitis, that involve the periodontium (the bone and soft tissues that support the teeth in the jaws). Gingivitis, an inflammation of the gums, is a very common condition. Periodontitis is also common and is a more severe condition that causes loss of bone that supports the teeth. It afflicts 15 to 20 per cent of the adult dentate (people with their natural teeth) population in Australia. ${ }^{2}$ Research over the last 30 years has described the role of bacterial plaque in causing periodontal disease, and controlled clinical trials suggest that periodontal treatment (such as scaling and cleaning of teeth) usually stabilises the condition and improves periodontal health. ${ }^{3,4}$ Thus, periodontal management, with an emphasis on bacterial plaque control, is an evidence-based intervention with established health outcomes.

A wide range of systemic conditions, ranging from the hormonal changes of puberty and pregnancy to disease 
entities involving immune dysfunction, connective tissue disease and malignancy, have manifestations in the mouth. The dynamics of the periodontium are a product of its circulation, hormonal changes and immune response mechanisms. Changes in systemic health that affect any of these factors can be reflected in changes in periodontal health. While this is well known, the main concerns of periodontics remain focused on oral causes, oral risks and oral remedies. Indeed, dental education, management and research have been limited by the dualistic notion that the oral cavity is separate from the rest of the body.

However, recent research suggests that periodontal diseases can influence systemic health through two mechanisms. The first is direct, by the pathogenic action of dental plaque bacteria that enter the bloodstream (bacteraemia). The second is indirect, by the distant effect of inflammatory mediators, such as cytokines, prostaglandins and serum antibodies, that are induced by periodontal disease.

\section{RECENT RESEARCH}

\section{Preterm birth}

Preterm birth is usually a result of rupture of membranes before 37 weeks gestation or preterm labour. The rate of preterm birth remains unchanged at approximately 6.3 per cent of all pregnancies in Australia despite improvements in antenatal care. Estimates are that onethird of premature births are of unknown causes. Approximately 75 per cent of spending on neonatal morbidity in Australia is as a result of preterm birth.

Maternal infection is associated with preterm labour. For example, infection in the maternal genito-urinary tract is associated with a higher risk of preterm birth. Periodontal disease, particularly gingivitis, is common among pregnant women. This is thought to be a result of an increased inflammatory response modulated by the effect of sex steroid hormones on the gums. ${ }^{5}$ The frequency of transient bacteraemias means there is a high risk of translocation of bacteria pathogens or the products of chronic periodontal infection, such as interleukin-1ß and prostaglandin $\mathrm{E}_{2}$, to other parts of the body.

For example, Fusobacterium nucleatum has been isolated from amniotic fluid among women with preterm labour but who had intact membranes. F. nucleatum is prevalent in the space between the gum and the tooth in humans, but not prevalent in the vaginal microflora. This suggests that the F. nucleatum infection in the amniotic fluid may have originated in the mouth. ${ }^{6}$

Recently, a case-control study of 124 mothers found that mothers of preterm babies had poor periodontal health when compared with mothers of normal birth-weight infants. $^{7}$ This association remained after controlling for factors such as smoking, alcohol consumption, age, race and level of prenatal care, which suggests that periodontal infection is associated with premature labour. This association has not been verified in an Australian population.

\section{Diabetes}

Type 2, or non-insulin-dependent diabetes (NIDDM), is the predominant form of diabetes in developed countries. Its prevalence is increasing in Australia because of greater awareness of the condition (leading to increased diagnosis and reporting), shifts in lifestyle patterns, an aging population and a higher prevalence of diabetes in some recent immigrant groups. Further, Aboriginal people and Torres Strait Islanders have the fourth highest prevalence rate of NIDDM in the world. ${ }^{8}$

Periodontal disease is a complication of diabetes. ${ }^{9}$ Numerous studies have shown that being diabetic is associated with a greater risk and severity of periodontal disease, particularly if the diabetes is poorly controlled. This susceptibility is linked to elevated blood glucose, altered protein synthesis, and changes in the microcirculation of the gums.

Conversely, recent evidence suggests that periodontal disease may cause poor control of diabetes. The Gila River Indian Community in Arizona has a prevalence of NIDDM of 50 per cent among its adults, the highest reported anywhere in the world. Recent analysis of periodontal data and blood sugars from this group showed that severe periodontitis was a risk for poor blood sugar control. ${ }^{10}$ Prospective controlled clinical trials in an Australian population could help to determine whether periodontal treatment has a beneficial effect on the stability of blood sugar control.

\section{Cardiovascular Disease}

Eating, brushing teeth, flossing and dental treatment may all lead to transient bacteraemias with bacteria that reside in the mouth, such as the streptococcus species. Bacteraemia is a particular risk for people with damaged heart valves, prosthetic valves and various cardiac anomalies who may develop bacterial endocarditis if bacterial vegetations form in parts of the heart with turbulent blood flow. Maintaining good dental health in these patients will reduce bacteraemias from everyday activities and reduce the need for dental interventions.

However, of broader public health concern is the suggestion that periodontitis is an independent risk factor for cardiovascular diseases (CVD) such as atherosclerosis, coronary thrombosis, ischaemic heart disease and myocardial infarction. The fact that the periodontal diseases and CVD share many risk factors, such as smoking, low socio-economic status, stress, hypertension 
and social isolation, complicates the study of the relationship between these diseases.

Two studies sponsored by the United States Department of Veterans Affairs - the Normative Aging Study and the Dental Longitudinal Study-permitted a prospective assessment of the effect of periodontal disease on CVD. The Normative Aging Study followed a group of 2280 male veterans from the greater Boston area who were living independently in the community and were healthy when they entered the study in 1961. The Dental Longitudinal Study started in 1968, and its 1221 participants were part of a subsample of the original Normative Aging Study population.

Comprehensive oral examinations were carried out at threeyear intervals. A range of variables was assessed, including alveolar bone loss as an indicator of periodontal disease. Mortality and morbidity from coronary heart disease (myocardial infarction, angina pectoris) and stroke was measured, along with risk factors such as cigarette smoking, blood pressure, cholesterol levels and body mass index.

Statistical analysis indicated a strong association between alveolar bone loss, stroke and cardiovascular disease. Periodontal disease was found to be a risk factor that was additional to other risk factors for cardiovascular disease and an independent risk factor for stroke.

A possible causative mechanism has been suggested from evidence emerging from animal laboratory studies. The viridans group of streptococci are found in dental plaque and do not usually cause dental disease. However, they can enter the circulation through the mucosa surrounding the teeth and assume a pathogenic role. Rabbits infused with Streptococcus sanguis to determine whether infection would affect cardiovascular health exhibited dosedependent changes in electrocardiograms, heart rate, blood pressure and cardiac contractility. ${ }^{12}$ Further, platelet aggregation and fibrin formation was found on heart valve vegetations of endocarditis, together with areas of myocardial infarction. This suggested that dental plaque bacteria could adversely affect cardiovascular health by stimulating thrombogenic events. Porphyromonas gingivalis, a gram-negative periodontal pathogen, can also bind onto atheromatous plaques and modify or promote their development as it, like $S$. sanguis, expresses an antigen that can induce platelet aggregation.

\section{IMPLICATIONS AND DIRECTIONS}

The evidence that oral health modifies systemic health has implications not only for the future direction of dental care, research and education in Australia, but also for the broader public health community. Dental health should be promoted not only for itself but also as an integral part of general health care. Indeed, a curriculum that acknowledges that oral health care is an integral part of general health care is being developed at the Faculty of Dentistry at the University of Sydney. Scant information is available about periodontal disease and systemic health in Australia, suggesting a need for appropriate, welldesigned and adequately funded research. Public health care in Australia can only benefit from recognising the relationship between periodontal disease and systemic health.

\section{REFERENCES}

1. Page RC. The pathobiology of periodontal diseases may affect systemic diseases: Inversion of a paradigm. Ann Periodontol 1998; 3: 108-120.

2. Barnard PD 1993, National Oral Health Survey Australia 1987-88, Department of Health, Housing, Local Government and Community Services, AGPS Press, Canberra.

3. Axelsson P, Lindhe J. Effect of controlled oral hygiene procedures on caries and periodontal disease in adults. JClin Periodontol 1978; 5: 133-151.

4. Suomi JD, Greene JC, Vermillion JR, et al. The effect of controlled oral hygiene procedures on the progression of periodontal disease in adults: Results after third and final year. J Periodontol 1971; 42: 152-160.

5. Mariotti A. Sex steroid hormones and cell dynamics in the periodontium. Critical Reviews in Oral Biology and Medicine 1994; 5: 27-53.

6. Hill GB. Preterm birth: Associations with genital and possibly oral microflora. Ann Periodontol 1998; 3: 222-232.

7. Offenbacher S, Katz V, Fertik G, et al. Periodontal infection as a possible risk factor for preterm low birth weight. $J$ Periodontol 1996; 67: 1103-1113.

8. McCarty DJ, Zimmet P, Dalton A, Segal L, Welborn TA. The rise and rise of diabetes in Australia, 1996: A review of statistics, trends and costs. Canberra: Diabetes Australia, 1996.

9. Löe H. Periodontal Disease. The sixth complication of diabetes mellitus. Diabetes Care 1993; 16: 329-334.

10. Taylor GW, Burt BA, Becker MP, et al. Severe periodontitis and risk for poor glycemic control in patients with non-insulindependent diabetes mellitus. J Periodontol 1996; 67: 10851093.

11. Beck J, Garcia R, Heiss G, Vokonas PS, Offenbacher S. Periodontal disease and cardiovascular disease. J Periodontol 1996; 67: 1123-1137.

12. Herzberg MC, Meyer MW. Effects of oral flora on platelets: Possible consequences in cardiovascular disease. J Periodontol 1996; 67: 1138-1142. W 